\title{
SOME DIFFERENTIAL EQUATIONS OF ELASTICITY AND THEIR LIE POINT SYMMETRY GENERATORS
}

\author{
Jozef BOCKO*, Iveta GLODOVÁ*, Pavol LENGVARSKÝ* \\ ${ }^{\star}$ Faculty of Mechanical Engineering, Department of Applied Mechanics and Mechatronics, \\ Technical University of Košice, Letná 9,04200 Košice, Slovakia \\ jozef.bocko@tuke.sk, iveta.glodova@tuke.sk, pavol.lengvarsky@tuke.sk
}

\begin{abstract}
The formal models of physical systems are typically written in terms of differential equations. A transformation of the variables in a differential equation forms a symmetry group if it leaves the differential equation invariant. Symmetries of differential equations are very important for understanding of their properties. It can be said that the theory of Lie group symmetries of differential equations is general systematic method for finding solutions of differential equations. Despite of this fact, the Lie group theory is relatively unknown in engineering community. The paper is devoted to some important questions concerning this theory and for several equations resulting from the theory of elasticity their Lie group infinitesimal generators are given.
\end{abstract}

Key words: Lie Groups, Symmetry, Differential Equations, Elasticity, Group Generator

\section{INTRODUCTION}

The group theory was discovered by Évariste Galois, who applied it to study of polynomial equations. The so-called finite groups were used as permutation groups and later the symmetry groups were applied in geometry. The idea of continuous groups was first used by Norwegian mathematician Sophus Lie for description of properties of differential equations. The application of continuous groups started systematically in his works and the terms Lie group and Lie algebra are used in honor of this great mathematician. The theory of Lie groups is applied in many different areas of mathematics, physics and engineering (Azad et al., 2010; Drew and Kloster, 1989; Olver, 1986; Sansour and Bednarczyk, 1995; Schwarz, 1982, 1984, 1988; Simo and Fox, 1989). In the field of physics work of German mathematician Emmy Noether, who found connection between symmetries of differential equations and the conservation laws, is very known. The paper describes Lie theory of symmetries of differential equations and for some equations resulting from the elasticity theory infinitesimal generators of their Lie groups are given. The generators have been found by program for symbolic manipulation. More details concerning Lie symmetries of differential equations can be found in Drew and Kloster (1989); Euler and Steeb (1992), Head (1993, 1996), Sansour and Bufler (1992).

\section{DIFFERENTIAL EQUATIONS AND LIE POINT GROUPS}

Starting point is the description of the system of partial differential equations:

$$
W_{v}\left(x_{i}, u^{a}, u_{i}^{a}, u_{i j}^{a}, \ldots, u_{i_{1}, \ldots, i_{k}}^{a}\right)=0
$$

$v=1, \ldots, N$, where $\mathbf{u}=\left(u^{1}, \ldots, u^{m}\right)$ are the functions depending on independent variables $\mathbf{x}=\left(x_{1}, \ldots, x_{n}\right)$ and:

$$
u_{i_{1}, \ldots, i_{n}}^{\alpha}=\frac{\partial^{i_{1}+\ldots+i_{n}} u^{\alpha}}{\partial x_{1}^{i_{1}} \ldots \partial x_{n}^{i_{n}}}
$$

are the partial derivatives.

Shortly, a Lie group is a group and a manifold at the same time. For any two points $a$ and $b$ in the manifold, there exists multiplication operation giving $a b$ and this group operation has a continuous structure of the manifold.

Change of independent and dependent variables can be represented by the finite transformations:

$$
\begin{aligned}
& \bar{x}_{i}=\phi_{i}(\mathbf{x}, \mathbf{u}, \varepsilon) \\
& \bar{u}^{\alpha}=\psi^{\alpha}(\mathbf{x}, \mathbf{u}, \varepsilon)
\end{aligned}
$$

where $\varepsilon \in \mathbb{R}$ is a parameter of a group. Expanding (3) by Taylor series at $\varepsilon=0$ gives relations:

$$
\begin{aligned}
& \bar{x}_{i}=x_{i}+\varepsilon \xi_{i}(\mathbf{x}, \mathbf{u})+o\left(\varepsilon^{2}\right) \\
& \bar{u}^{\alpha}=u^{\alpha}+\varepsilon \eta^{\alpha}(\mathbf{x}, \mathbf{u})+o\left(\varepsilon^{2}\right)
\end{aligned}
$$

with the substitutions:

$$
\begin{aligned}
\xi_{i} & =\left.\frac{\partial \phi_{i}(\mathbf{x}, \mathbf{u}, \varepsilon)}{\partial \varepsilon}\right|_{\varepsilon=0} \\
\eta^{\alpha} & =\left.\frac{\partial \psi^{\alpha}(\mathbf{x}, \mathbf{u}, \varepsilon)}{\partial \varepsilon}\right|_{\varepsilon=0}
\end{aligned}
$$

The value $\varepsilon=0$ is the identity element of the group. Equations (5) allow us to write infinitesimal generator, or Lie point symmetry vector field by relation:

$$
\mathbf{U}=\xi_{i} \frac{\partial}{\partial x_{i}}+\eta^{\alpha} \frac{\partial}{\partial u^{\alpha}}
$$

The transformation of partial derivatives influences the socalled k-th prolongation of a vector field $\mathrm{U}$ :

$$
\mathbf{U}^{(k)}=\mathbf{U}+\zeta_{i}^{\alpha} \frac{\partial}{\partial u_{i}^{\alpha}}+\ldots+\zeta_{i_{1} \ldots i_{k}}^{\alpha} \frac{\partial}{\partial u_{i_{1} \ldots i_{k}}^{\alpha}}
$$


where the functions $\xi_{i_{1} \ldots i_{k}}^{\alpha}$ describe the transformations of partial derivatives of order $k$. The functions $\xi_{i_{1} \ldots i_{k}}^{\alpha}$ are determined according to relations:

$\zeta_{i}^{\alpha}=\mathrm{D}_{i}\left(\eta^{\alpha}\right)-u_{s}^{\alpha} \mathrm{D}_{i}\left(\xi_{s}\right)$

and:

$\zeta_{i_{1} \ldots i_{k}}^{\alpha}=\mathrm{D}_{i_{k}}\left(\zeta_{i_{1} \ldots i_{k-1}}^{\alpha}\right)-u_{i_{1} \ldots i_{k-1}}^{\alpha},{ }_{s} \mathrm{D}_{i_{k}}\left(\xi_{s}\right)$

where:

$\mathrm{D}_{i}=\frac{\partial}{\partial x_{i}}+u_{i}^{\alpha} \frac{\partial}{\partial u^{\alpha}}+u_{k i}^{\alpha} \frac{\partial}{\partial u_{k}^{\alpha}}+u_{k l i}^{\alpha} \frac{\partial}{\partial u_{k l}^{\alpha}} \ldots$

is the operator of total differentiation with respect to the independent variable $x_{i}$. The group is symmetry of equation system (2) if and only if the invariant surface condition (symmetry condition):

$\mathbf{U}^{(k)} \Omega_{v}=0$

is satisfied, where all $\Omega_{v}=0, v=1, \ldots, N$. The components $\xi_{\mathrm{i}}$ and $\eta^{\alpha}$ of the infinitesimal generator $U$ are determined from equations (11).

The infinitesimal generator and Lie group are connected by relation:

$$
(\overline{\mathbf{x}}, \overline{\mathbf{u}})=g_{\varepsilon} \cdot(\mathbf{x}, \mathbf{u})=\left(\boldsymbol{\phi}_{\varepsilon}(\mathbf{x}, \mathbf{u}), \boldsymbol{\psi}_{\varepsilon}(\mathbf{x}, \mathbf{u})\right)=e^{\varepsilon \mathbf{U}}(\mathbf{x}, \mathbf{u})
$$

Here, the Greek letter $\varepsilon$ is reserved for the parameter of the group and accordingly it does not represent partial differentiation. The function $\mathbf{f}$ undergoes the transformation by the group element $g_{\varepsilon}$ in accordance with relation:

$$
\overline{\mathbf{u}}=\overline{\mathbf{f}}_{\varepsilon}(\overline{\mathbf{x}})=\left(g_{\varepsilon} \mathbf{f}\right)(\overline{\mathbf{x}})=\left[\boldsymbol{\psi}_{\varepsilon} \circ(\mathbf{1} \times \mathbf{f})\right] \circ\left[\boldsymbol{\phi}_{\varepsilon} \circ(\mathbf{1} \times \mathbf{f})\right]^{-1}(\overline{\mathbf{x}})
$$

where 1 represents the identity function $\mathbf{1}(\mathbf{x})=\mathbf{x}$.

Calculation of the Lie vector fields from equations (11) is tedious work. It involves a large amount of symbolic calculations that is better done by computer. Fortunately, different packages in computer algebra systems exist implementing Lie symmetry computations (Champagne et al., 1991; Lie, 1891, 1896; Vu et al., 2012).

\section{THICK-WALLED PIPE}

Thick-walled pressure vessels and pipes have many applications in engineering practice. Differential equation describing radial stress in a pipe is:

$\frac{d^{2} \sigma(r)}{\partial r^{2}}+\frac{3}{r} \frac{d \sigma(r)}{d r}=0$

where $\sigma(r)$ is the radial stress and $r$ is the independent variable representing radius. As was mentioned above, there are number programs for solving determining equations of the vector fields resulting from equation (11). They work under different systems for symbolic manipulations, e.g. Reduce, Mathematica, Maple, and so on (Champagne et al., 1991; Lie, 1891, 1986; Vu et al., 2012). In our case we have used DESOLVII [19] working under system Maple. For equation (14) the program DESOLVII gives us the following Lie symmetry vector fields:

$$
\begin{aligned}
& \mathbf{U}_{1}=-2 \sigma^{2} \frac{\partial}{\partial \sigma}+\sigma r \frac{\partial}{\partial r} \\
& \mathbf{U}_{2}=\frac{1}{r^{2}} \frac{\partial}{\partial \sigma} \\
& \mathbf{U}_{3}=r \frac{\partial}{\partial r} \\
& \mathbf{U}_{4}=r^{3} \frac{\partial}{\partial r} \\
& \mathbf{U}_{5}=-\frac{2 \sigma}{r^{2}} \frac{\partial}{\partial \sigma}+\frac{1}{r} \frac{\partial}{\partial r} \\
& \mathbf{U}_{6}=\sigma \frac{\partial}{\partial \sigma} \\
& \mathbf{U}_{7}=\sigma r^{3} \frac{\partial}{\partial r} \\
& \mathbf{U}_{8}=\frac{\partial}{\partial \sigma}
\end{aligned}
$$

These vector fields are infinitesimal generators of Lie groups of symmetries of differential equation describing radial stress in thick-walled pipe. The corresponding Lie groups can be established from vector fields according to equation (12).

\section{AXISYMMETRIC PLATE}

Differential equation for deformation of axisymmetric plate loaded by uniformly distributed load can be written as:

$$
\frac{d^{3} w(r)}{d r^{3}}+\frac{1}{r} \frac{d^{2} w(r)}{d r^{2}}-\frac{1}{r^{2}} \frac{d w(r)}{d r}=Q
$$

where $w(r)$ is the deflection of the plate at the radius $r$, the constant $Q=\frac{T}{D}$ depends on the constant uniformly distributed load $T$ and the constant plate stiffness $D$. The plate stiffness is

$$
D=\frac{E h^{3}}{12\left(1-v^{2}\right)}
$$

where $E$ and $v$ is Young modulus and Poisson ratio of plate material, respectively; $h$ is the plate thickness.

Here again the program DESOLVII has been used for solution of determining equations that correspond to the equation (16). Resulting infinitesimal generators are:

$$
\begin{aligned}
& \mathbf{U}_{1}=Q r^{3} \frac{\partial}{\partial w}+3 r \frac{\partial}{\partial r} \\
& \mathbf{U}_{2}=r^{2} \frac{\partial}{\partial w} \\
& \mathbf{U}_{3}=\left(Q r^{3}-9 w\right) \frac{\partial}{\partial w} \\
& \mathbf{U}_{4}=\ln (r) \frac{\partial}{\partial w} \\
& \mathbf{U}_{5}=\frac{\partial}{\partial w}
\end{aligned}
$$




\section{VIBRATING BEAM WITH DAMPING}

Differential equation for vibration of a beam with damping is:

$$
\frac{\partial^{4} u(x, \mathrm{t})}{\partial x^{4}}+\frac{k^{4}}{\alpha^{2}} \frac{\partial^{2} u(x, \mathrm{t})}{\partial t^{2}}+\mu \frac{\partial^{5} u(x, \mathrm{t})}{\partial x^{4} \partial t}=0
$$

where $u(x, \mathrm{t})$ is the deflection of beam at the position $x$ and the time instant t. Constant $\frac{k^{4}}{\alpha^{2}}=\frac{\rho A}{E J}$ depends on the material density $\rho$, Young modulus $E$, the cross-section area $A$ and moment of inertia of the beam's cross-section $J . \mu$ is the coefficient of internal damping of material.

Determining equations (11) for differential equation (19) have been solved by program DESOLVII. Resulting infinitesimal generators are:

$$
\begin{aligned}
& \mathbf{U}_{1}=u \frac{\partial}{\partial u} \\
& \mathbf{U}_{2}=\frac{\partial}{\partial t} \\
& \mathbf{U}_{3}=\frac{\partial}{\partial x} \\
& \mathbf{U}_{4}=f(x, t) \frac{\partial}{\partial u}
\end{aligned}
$$

Here, function $F(x, t)$ is any solution of equation (19). The vector field $U_{2}$ represents simple shifting in time, the vector $\mathrm{U}_{3}$ shifting in the direction $\mathrm{x}$.

\section{MEMBRANE}

Differential equation of a stretched membrane in the plane $\mathrm{x}, \mathrm{y}$ which is loaded by the constant pressure $\mathrm{p}$ can be written in the form:

$$
\frac{\partial^{2} w}{\partial x^{2}}+\frac{\partial^{2} w}{\partial y^{2}}=-\frac{p}{S}
$$

where $w(\mathrm{x}, \mathrm{y})$ is the deflection of a membrane perpendicular to the membrane plane and $S$ is the tension force per unit length of the membrane. Here, the infinitesimal generators of equation (21) are:

$$
\begin{aligned}
& \mathbf{U}_{1}=w \frac{\partial}{\partial w} \\
& \mathbf{U}_{2}=g_{1}(x, y) \frac{\partial}{\partial x} \\
& \mathbf{U}_{3}=g_{2}(x, y) \frac{\partial}{\partial y} \\
& \mathbf{U}_{4}=g_{3}(x, y) \frac{\partial}{\partial w}
\end{aligned}
$$

The functions $g_{1}(x, y), g_{2}(x, y), g_{3}(x, y)$ represent any solution of differential equation (21).

\section{PLATE ON ELASTIC FUSS-WINKLER FOUNDATION}

Differential equation describing deformation of a plate on elastic Fuss-Winkler foundation is:

$$
D\left(\frac{\partial^{4} w}{\partial x^{4}}+2 \frac{\partial^{4} w}{\partial x^{2} \partial y^{2}}-\frac{\partial^{4} w}{\partial y^{4}}\right)+K w(x, y)=p(x, y)
$$

where $\mathrm{w}(\mathrm{x}, \mathrm{y})$ is the deflection of the plate at the point with the coordinates $\mathrm{x}, \mathrm{y}$, constant $\mathrm{D}$ is the plate stiffness given by equation (17), $\mathrm{K}$ is the coefficient of subgrade reaction and $p(x, y)$ is the pressure acting on the plate. The Lie group generators of given differential equation are:

$$
\begin{aligned}
& \mathbf{U}_{1}=\frac{\partial}{\partial x} \\
& \mathbf{U}_{2}=\frac{\partial}{\partial y} \\
& \mathbf{U}_{3}=x \frac{\partial}{\partial y}-y \frac{\partial}{\partial x} \\
& \mathbf{U}_{4}=w \frac{\partial}{\partial w} \\
& \mathbf{U}_{5}=h(x, y) \frac{\partial}{\partial w}
\end{aligned}
$$

Here, $\mathrm{h}(\mathrm{x}, \mathrm{y})$ is any solution of differential equation (23). The vector fields $U_{1}, U_{2}$ represent simple shifting along the coordinate $\mathrm{x}$ and $\mathrm{y}$ respectively.

Let us now compute less trivial example of transformation that belong to the Lie vector:

$$
\mathbf{U}_{3}=x \frac{\partial}{\partial y}-y \frac{\partial}{\partial x}
$$

For the transformation of the independent variable $\mathrm{x}$ we have:

$$
\begin{aligned}
& \mathbf{U}_{3}(x)=-y \\
& \mathbf{U}_{3}^{2}(x)=-\mathbf{U}_{3}(y)=-x \\
& \mathbf{U}_{3}^{3}(x)=-\mathbf{U}_{3}(x)=y \\
& \mathbf{U}_{3}^{4}(x)=\mathbf{U}_{3}(y)=x \\
& \vdots \\
& \bar{x}=x+\varepsilon(-y)+\frac{\varepsilon^{2}}{2 !}(-x)+\frac{\varepsilon^{3}}{3 !}(y)+\frac{\varepsilon^{4}}{4 !}(x)+\ldots= \\
& \quad x \cos \varepsilon-y \sin \varepsilon
\end{aligned}
$$

For the independent variable y we have similar relation:

$$
\begin{aligned}
& \mathbf{U}_{3}(y)=x \\
& \mathbf{U}_{3}^{2}(y)=-y \\
& \mathbf{U}_{3}^{3}(y)=-x \\
& \mathbf{U}_{3}^{4}(y)=y
\end{aligned}
$$

$$
\begin{aligned}
\bar{y}= & y+\varepsilon(x)+\frac{\varepsilon^{2}}{2 !}(-y)+\frac{\varepsilon^{3}}{3 !}(-x)+\frac{\varepsilon^{4}}{4 !}(y)+\ldots= \\
& y \cos \varepsilon+x \sin \varepsilon
\end{aligned}
$$

We see that vector $U_{3}$ is connected with the rotation of variables in the plain $x y$. 
All transformation groups that belong to vectors (24) are:

$$
\begin{aligned}
& G_{1}:(x, y, w) \mapsto(x+\varepsilon, y, w) \\
& G_{2}:(x, y, w) \mapsto(x, y+\varepsilon, w) \\
& G_{3}:(x, y, w) \mapsto(x \cos \varepsilon-y \sin \varepsilon, x \sin \varepsilon+y \cos \varepsilon, w) \\
& G_{4}:(x, y, w) \mapsto\left(x, y, \mathrm{e}^{\varepsilon} w\right) \\
& G_{5}:(x, y, w) \mapsto(x, y, w+\varepsilon h(x, y))
\end{aligned}
$$

The groups (28) represent transformations that convert solutions of differential equation (23) into new solutions of the same equation.

\section{CONCLUSIONS}

The notion of Lie group is very important in the current mathematics and physics. The paper analyzes differential equations resulting from different branches of elasticity theory from the point of view of their symmetries. Lie vectors of corresponding Lie groups symmetries of differential equations have been computed by computer program DESOLVII. Infinitesimal generators of Lie group symmetries give us additional information that is not visible during classical solutions of differential equations.

\section{REFERENCES}

1. Azad H., Mustafa M. T., Arif A. F. M. (2010), Analytic Solutions of Initial-Boundary-Value Problems of Transient Conduction Using Symmetries, Applied Mathematics and Computation, Vol. 215, 41324140.

2. Bluman G. W., Cole J. D. (1974), Similarity Methods for Differential Equations, Springer-Verlag, New York, 1974.

3. Champagne B., Hereman W., Winternitz P. (1991), The Computer Calculation of Lie Point Symmetries of Large Systems of Differential Equations, Computer Physics Communications, Vol. 66, 319-340.

4. Drew M. S., Kloster S. (1989), Lie Group Analysis and Similarity Solutions for the Equation $\frac{\partial^{2} u}{\partial x^{2}}+\frac{\partial^{2} u}{\partial y^{2}}+\frac{\partial^{2}\left(e^{u}\right)}{\partial z^{2}}=0$, Nonlinear Analysis, Theory Methods Applications, Vol. 13, No. 5, 1989, 489505.

5. Euler N., Steeb W.-H. (1992), Continuous Symmetries, Lie Algebras and Differential Equations, Brockhaus AG, Mannheim.
6. Head A. K. (1993), LIE a PC Program for Lie Analysis of Differential Equations, Computer Physics Communications, Vol. 71, 241-248.

7. Head A. K. (1996), Instructions for Program LIE ver. 4.5, CSIRO, Australia.

8. Lie S. (1891), Vorlesungen über Differentialgleichungen mit bekannten infinitesimalen Transformationen, Teubner, Leipzig.

9. Lie S. (1896), Geometrie der Berührungstransformationen, Teubner, Leipzig.

10. Olver P. J. (1986), Applications of Lie Groups to Differential Equations, Springer-Verlag, New York.

11. Sansour C., Bednarczyk H. (1991), Shells at Finite Rotations with Drilling Degrees of Freedom, Theory and Finite Element Formulation, In: Glowinski R., Ed., Computing Methods in Applied Sciences and Engineering, Nova Sci. Publish., New York, 163-173.

12. Sansour C., Bednarczyk H. (1995), The Cosserat Surface as a Shell Model, Theory and Finite-Element Formulation, Computer Methods in Applied Mechanical Engineering, Vol. 120, 1-32.

13. Sansour C., Bufler H. (1992), An Exact Finite Rotation Shell Theory, its Mixed Variational Formulation, and its Finite Element Implementation, International Journal for Numerical Methods in Engineering, Vol. 34, 73-115.

14. Schwarz F. (1982), Symmetries of the Two-Dimensional Kortewegde Vries Equation, Journal of the Physical Society of Japan, Vol. 51, No. 8, 2387-2388.

15. Schwarz F. (1984), Lie Symmetries of the von Kármán Equations, Computer Physics Communications, Vol. 31, 113-114.

16. Schwarz F. (1988), Symmetries of Differential Equations from Sophus Lie to Computer Algebra. SIAM Review, Vol. 30, No. 3, 450481.

17. Sherring J., Head A. K., Prince G. E. (1997), DIMSYM and LIE: Symmetry Determination Packages. Algorithms and Software for Symbolic Analysis of Nonlinear Systems, Mathematical and Computer Modelling, Vol. 25, No. 8-9, 153-164.

18. Simo J. C., Fox D. D. (1989), On a Stress Resultant Geometrically Exact Shell Model, Part I.: Formulation and Optimal Parametrization, Computer Methods in Applied Mechanics and Engineering, Vol. 72, 267-304.

19. Vu K. T., Jefferson G. F., Carminati J. (2012), Finding Higher Symmetries of Differential Equations Using the MAPLE Package DESOLVII, Computer Physics Communications, Vol. 183, No. 4, 1044-1054.

The work has been accomplished under the research project of Slovak grant agency VEGA 1/1205/12 - Numerical modeling of mechatronic systems. 